\title{
Effects of Hemoadsorption with CytoSorb during Severe Rhabdomyolysis
}

\author{
Hannah C. Daum ${ }^{a}$ Bernhard M.W. Schmidt ${ }^{b}$ Lars Christian Napp ${ }^{a}$ \\ ${ }^{a}$ Cardiac Arrest Center, Department of Cardiology and Angiology, Hannover Medical School, Hannover, Germany; \\ ${ }^{b}$ Department of Nephrology and Hypertension, Hannover Medical School, Hannover, Germany
}

Dear Editor,

Severe rhabdomyolysis occurs due to various causes and injuries and can result in several potentially life-threatening complications $[1,2]$, of which acute kidney injury (AKI) is one of the most important adverse events. The pathomechanism of AKI in rhabdomyolysis is complex, and different mechanisms such as direct tubular damage by oxidative injury, tubular obstruction by precipitated Tamm-Horsfall protein-myoglobin complexes, and hypovolemia-associated renal vasoconstriction play a role $[3,4]$. Of note, in most of these processes, myoglobin released by injured muscle is essentially and directly involved $[3,5]$. In contrast, creatine kinase $(\mathrm{CK})$ probably plays no mechanistic role in $\mathrm{AKI}$, and its value as a marker for AKI remains controversial $[6,7]$. In addition, other factors contribute to renal damage in patients with rhabdomyolysis, such as surgery, the underlying condition, or trauma. Beyond AKI, potassium and calcium disturbance, metabolic acidosis, and disseminated intravascular coagulation may arise, also depending on the cause of muscle injury [1].

Management of rhabdomyolysis intends to increase the clearance of myoglobin, to treat hypovolemia, and to balance electrolyte and $\mathrm{pH}$ changes, for preventing the complications mentioned above [2]. In addition to myoglobin, there may be other currently unknown mediators of damage released from muscle to peripheral blood, which could be removed by extracorporeal therapies. In contrast, the therapeutic value of removing CK during rhabdomyolysis is unclear. Dialysis with high cutoff membranes is frequent-

karger@karger.com

(c) 2020 S. Karger AG, Basel

www.karger.com/bpu

Karger ly used in the management of rhabdomyolysis $[8,9]$. However, in clinical practice, this approach is often not sufficient, and may be associated with unwanted loss of other molecules from the circulation. In this context, we read the report of Dilken et al. [10] with great interest.

The authors describe the case of a 56-year-old male patient who experienced a severe crush injury to the lower extremities and the abdominal wall, which led to traumatic rhabdomyolysis. Continuous veno-venous hemodialysis was initiated with a high cutoff membrane (EMIC-2, Fresenius Medical Care, Bad Homburg, Germany). This was associated with stabilization of myoglobin levels in peripheral blood in the presence of further increasing CK activity. The authors added a CytoSorb ${ }^{\circledR}$ hemoadsorption device to the hemodialysis circuit and observed a rather rapid decrease of CK activity and slowly decreasing myoglobin levels. This observation raised questions to us.

Hemoadsorption with CytoSorb ${ }^{\circledR}$ is increasingly used as stand-alone hemoperfusion, with various renal replacement circuits or with extracorporeal membrane oxygenation [11]. It adsorbs small hydrophobic molecules up to a size of around 55-60 kD. Myoglobin has a mass of $17 \mathrm{kD}$ [12-14] and is effectively adsorbed by CytoSorb ${ }^{\circledR}$, which resulted in approval of the device for conditions with increased myoglobin levels such as rhabdomyolysis [15]. CK in its dimeric form, however, is certainly too large to be adsorbed by CytoSorb ${ }^{\circledR}$ and to be dialyzable even with a high cutoff membrane. CK is a dimer of two $43-\mathrm{kD}$ subunits [14, $16,17]$, and the predominant type in peripheral blood is the 
MM-isoenzyme, in particular during rhabdomyolysis [18]. Although we cannot exclude that circulating CK-M monomers are adsorbed by the device, this would very likely not explain the rapid decrease of CK activity, which was observed already $4 \mathrm{~h}$ after initiation of hemoadsorption. Moreover, myoglobin initially increased and then only slowly decreased after installation of CytoSorb ${ }^{\circledR}$, further arguing against the hypothesis that CytoSorb ${ }^{\circledR}$ was responsible for the strong drop of CK activity during that phase. It would be helpful to know when exactly surgical procedures were performed, as it appears likely that dialysis and hemoadsorption were interrupted by surgery and that surgery modified rhabdomyolysis burden. Furthermore, we were wondering if and when the dialysate and membranes were replaced during the time period displayed in the graphs. For interpretation of the results, it would be important to know if a new dialysis unit was used with installation of CytoSorb ${ }^{\circledR}$. The authors further state that saturation of the adsorber was noticed, and we are curious how saturation of the device was determined. Moreover, did the authors observe changes in other factors known to be adsorbed by CytoSorb ${ }^{\circledR}$, such as interleukin-6?

In summary, CytoSorb ${ }^{\circledR}$ adsorbs myoglobin with reportedly high efficacy. In addition, the device may adsorb other known and unknown molecules, which potentially play a role during pathogenesis of rhabdomyolysis. We fully agree with the authors that hemoadsorption is a potentially attractive adjunctive treatment for this condition, in addition to volume therapy, forced diuresis, and dialy- sis if indicated. However, we doubt that CytoSorb ${ }^{\circledR}$ lowers CK activity by adsorbing the enzyme, at least in its dimeric form, and that lowering CK per se is of clinical importance. To the best of our knowledge, there is no publication directly proving adsorption of CK by CytoSorb ${ }^{\circledR}$. Therefore, we suggest measuring levels of target molecules before and after the adsorber also in clinical settings, in order to provide definitive evidence of substance removal.

\section{Disclosure Statement}

There are no conflicts of interest regarding the content of this letter. Outside the present work, H.C.D. has no conflicts of interest to declare. B.M.W.S. received lecture honoraria from Fresenius Medical Care. L.C.N. received lecture and consulting honoraria and research support from Cytosorbents. Other relationships beyond the topic of this letter exist.

\section{Funding Sources}

There was no funding involved in this letter.

\section{Author Contributions}

H.C.D. drafted the manuscript, B.M.W.S. revised the manuscript for important intellectual content, and L.C.N. designed, supervised, and revised the manuscript.

\section{References}

1 Torres PA, Helmstetter JA, Kaye AM, Kaye AD. Rhabdomyolysis: pathogenesis, diagnosis, and treatment. Ochsner J. 2015;15(1):58-69.

2 Vanholder R, Sever MS, Erek E, Lameire N. Rhabdomyolysis. J Am Soc Nephrol. 2000; 11(8):1553-61.

3 Bosch X, Poch E, Grau JM. Rhabdomyolysis and acute kidney injury. N Engl J Med. 2009; 361(1):62-72.

4 Petejova N, Martinek A. Acute kidney injury due to rhabdomyolysis and renal replacement therapy: a critical review. Crit Care. 2014; 18(3):224.

5 Holt S, Moore K. Pathogenesis of renal failure in rhabdomyolysis: the role of myoglobin. Exp Nephrol. 2000;8(2):72-6.

6 Simpson JP, Taylor A, Sudhan N, Menon DK, Lavinio A. Rhabdomyolysis and acute kidney injury: creatine kinase as a prognostic marker and validation of the McMahon Score in a 10-year cohort: a retrospective observational evaluation. Eur J Anaesthesiol. 2016;33(12):906-12.
7 Talving P, Karamanos E, Skiada D, Lam L, Teixeira PG, Inaba K, et al. Relationship of creatine kinase elevation and acute kidney injury in pediatric trauma patients. J Trauma Acute Care Surg. 2013;74(3):912-6.

8 Ronco C. Extracorporeal therapies in acute rhabdomyolysis and myoglobin clearance. Crit Care. 2005;9(2):141-2.

9 Sorrentino SA, Kielstein JT, Lukasz A, Sorrentino JN, Gohrbandt B, Haller H, et al. High permeability dialysis membrane allows effective removal of myoglobin in acute kidney injury resulting from rhabdomyolysis. Crit Care Med. 2011;39(1):184-6.

10 Dilken O, Ince C, van der Hoven B, Thijsse S, Ormskerk P, de Geus HRH. Successful reduction of creatine kinase and myoglobin levels in severe rhabdomyolysis using extracorporeal blood purification (CytoSorb ${ }^{\circ}$ ). Blood Purif. 2020:1-5.

11 Napp LC, Ziegeler S, Kindgen-Milles D. Rationale of hemoadsorption during extracorporeal membrane oxygenation support. Blood Purif. 2019;48(3):203-14.
12 Perkoff GT, Hill RL, Brown DM, Tyler FH. The characterization of adult human myoglobin. J Biol Chem. 1962;237:2820-7.

13 Available from: https://www.uniprot.org/uniprot/P02144. Accessed 2020 Apr 21.

14 UniProt C. UniProt: a worldwide hub of protein knowledge. Nucleic Acids Res. 2019; 47:D506-D515.

15 Cytosorbents Inc.: Instructions for use, Document Number 17-0071-14.

16 Available from: https://www.uniprot.org/uniprot/P06732. Accessed 2020 Apr 21.

17 Wood TD, Chen LH, White CB, Babbitt PC, Kenyon GL, McLafferty FW. Sequence verification of human creatine kinase $(43 \mathrm{kDa})$ isozymes by high-resolution tandem mass spectrometry. Proc Natl Acad Sci U S A. 1995; 93(21):12051-5.

18 Brancaccio P, Lippi G, Maffulli N. Biochemical markers of muscular damage. Clin Chem Lab Med. 2010;48(6):757-67.
Does CytoSorb Really Clear Creatine Kinase? 